\title{
Structure of TiN/CrN Interface in Nanolaminate Coatings with Enhanced Mechanical and Tribological Properties
}

\author{
Sergey Yarmolenko ${ }^{1}$, Manohar Konchady ${ }^{2}$, Sudheer Neralla ${ }^{3}$, Alex Kvit ${ }^{4}$, Zhigang Xu ${ }^{1}$ and Jag \\ Sankar ${ }^{1 *}$ \\ 1. North Carolina A\&T State University, Mechanical Engineering, Greensboro, NC USA \\ 2. Intel Corporation, Phoenix, AZ USA \\ 3. Vishay Precision Group, Wendell, NC USA \\ 4. University of Wisconsin-Madison, Materials Science and Engineering, Madison, WI USA \\ * Corresponding Author: sankar@ncat.edu
}

Nanolaminates usually consist of alternating nano-layers of two or more different materials with unlike physical and mechanical properties, together forming new tailored-function materials with a combination of desired properties of its components, or better. This makes a principal difference from the traditional bulk multilayer coatings, when each material layer played a designated functional role. As the layer thickness approaches nanometer range, a significant increase in the volume fraction of grain boundaries leads to drastic changes in physical and chemical properties of the materials, often resulting in better hardness, toughness, residual stress, and thermal stability [1]. The results of previous studies clearly show that mechanical, thermal, and tribological properties of hard thin films can significantly be optimized by well-designed microstructures. Nano-multilayer coatings of transition metal nitrides, are an emerging class of superhard materials [2-4]. Single-layer TiN and CrN coatings are established tribological coatings. Previously we reported excellent tribolodical properties of $\mathrm{TiN} / \mathrm{CrN}$ multilayers [5-7] produced by reactive magnetron sputtering technique. We used custom-designed a state-of- the-art multifunctional magnetron sputtering deposition system (AJA International Inc.) allowing to simultaneously co-sputter materials from up to four targets with unlimited number of deposited layers, utilizing DC, RF, pulsed DC, balanced, unbalanced, and reactive sputtering techniques at temperatures from -200 to $800^{\circ} \mathrm{C}$. Another advances features are the ultra-high base vacuum, programmable reactive gas delivery of up to three reactive gases, substrate rotation, tunable gun positions, quadrupole mass-spectrometric residual gas analyzer.

In this study we present some details of TiN/CrN interface structure in nanolaminates and connected these observations them with mechanical/tribological properties on nanolaminates reported earlier [5-7]. Structure nanolaminates is shown in Figure 1. Optimal conditions of nanolaminate growth include $300^{\circ} \mathrm{C}$ substrate temperature and 5W RF-bias. Role of the bias indicate that improvement of film quality and their properties require additional energy not only for activation of reaction between $\mathrm{Cr}$ and $\mathrm{Ti}$ atoms ith nitrogen, but also some activation necessary for proper positioning of $\mathrm{TiN}$ and $\mathrm{CrN}$ molectules. Figure 2 shows that TiN/CrN nanolaminates have significant improvement in hardness and abrasive properties compared with single material layers with similar thickness (shown as horizontal lines). Property improvement become more pronounced as bi-layer thickness decreases and at layer thickness of each material $2 \mathrm{~nm}$, nanolaminate become superhard coatings with extremely high hardness $32 \mathrm{GPa}$ and have very high scratch resistance/roughness. These observations indicate important role of interface between TiN and CrN crystalline layers, which should provide certain separation between layers (like grain boundary in nanocrystalline materials) and moderate shear strength of interphase. TEM images of nanolaminate show that nanolaminate exhibit continuous columnar structure across layers seen at low magnification (Figure 3a) low magnification image is typical for all studied nanolaminates with bilayer 
thicknesses $10,20,40,100$ and $200 \mathrm{~nm}$ ) which is not really have crystal alignment through interface at close-up view (Figure 3b). Lattice mismatch between TiN and $\mathrm{CrN}$ is relatively small (4.24 $\AA$ and $4.14 \AA$ or just $2.4 \%$ ) but detail analysis of SAED showed that $\mathrm{TiN}$ and $\mathrm{CrN}$ create unusual stacking structure rotated on $45^{\circ}$ (Figure $3 \mathrm{c}$ ).

References:

[1] M. Stueber et al., Journal of Alloys and Compounds 483(1-2) (2009), p. 321.

[2] HC Barshilia, A Jain and KS Rajam, Vacuum 72(3) (2003), p. 241.

[3] HC Barshilia, KS Rajam and DVS Rao, Surface \& Coatings Technology 200(14-15) (2006), p. 4586.

[4] YM Zhou et al., Wear 236(1-2) (1999), p. 159.

[5] MS Konchady et al., Proceedings of the Asme International Mechanical Engineering Congress and Exposition (2007): Design and Manufacturing New York: Amer Soc Mechanical Engineers (2008) p. 313.

[6] MS Konchady et al., IMECE2009: Proceedings of the Asme International Mechanical Engineering Congress and Exposition New York: Amer Soc Mechanical Engineers (2010) p. 55-59.

[7] MS Konchady et al., International Journal of Surface Science and Engineering 2(6) (2008), p. 439.

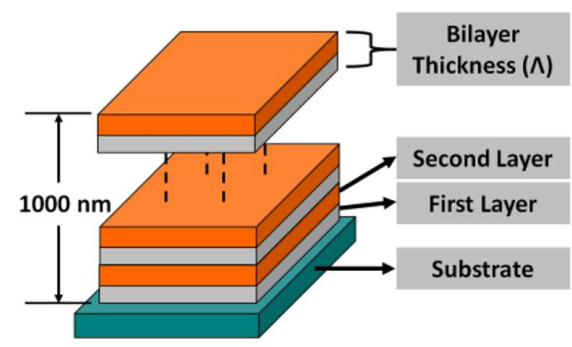

Bilayer thicknesses: 5, 10, $20,40,100,200,500$ and $1000 \mathrm{~nm}$

Figure 1. TiN/CrN Nanolaminates

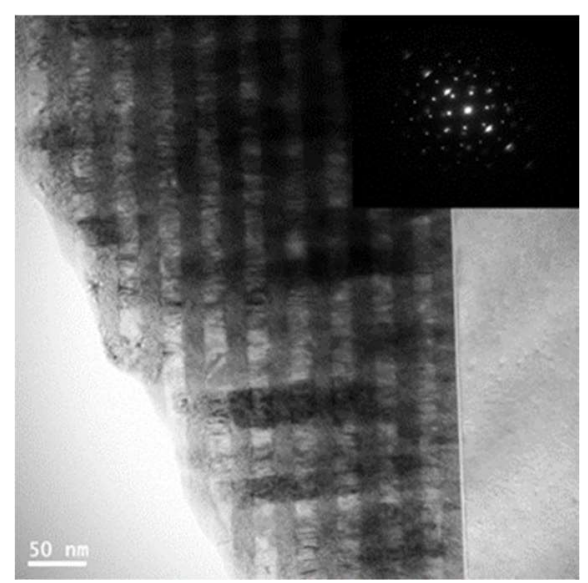

(a)

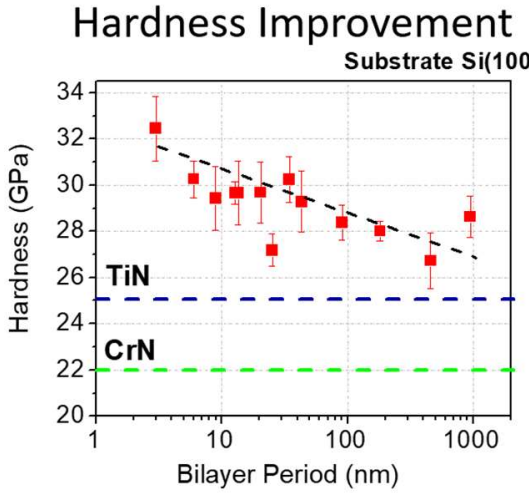

Abrasion Improvement

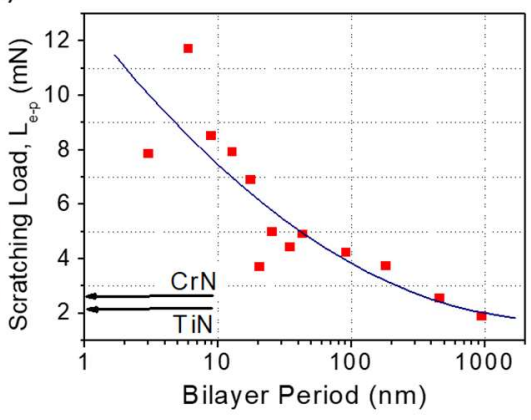

Figure 2. Hardness and abrasion properties of TiN/CrN nanolaminates

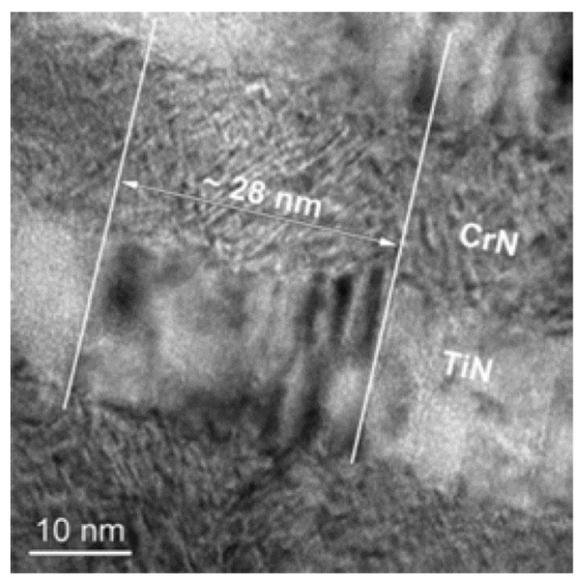

(b)

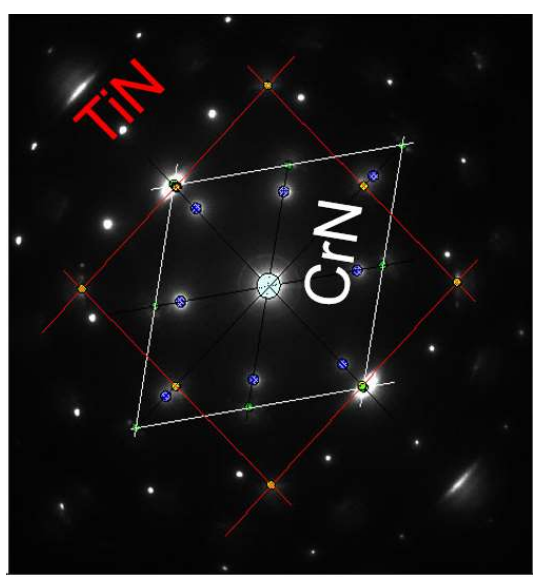

(c)

Figure 3. TEM image of TiN/CrN nanolaminates: (a) example of $40 \mathrm{~nm}$ bi-layer thickness nanolaminate, (b) hither magnification of layers, (c) analysis of SAED show twisted structure of TiN/CrN interface. 